\title{
Lower regional grey matter in alcohol use disorders: evidence from a voxel-based meta-analysis
}

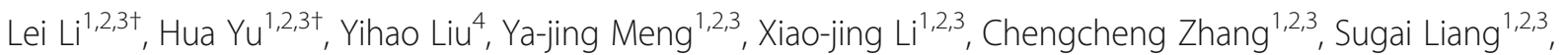
Ming-li Li, ${ }^{1,2,3}$, Wanjun Guo ${ }^{1,2,3}$, QiangWang ${ }^{1,2,3}$, Wei Deng ${ }^{1,2,3}$, Xiaohong Ma ${ }^{1,2,3}$, Jeremy Coid ${ }^{1,2,3}$ and Tao $\mathrm{Li}^{1,2,3^{*}}$ (1)

\begin{abstract}
Background: Previous research using whole-brain neuroimaging techniques has revealed structural differences of grey matter (GM) in alcohol use disorder (AUD) patients. However, some of the findings diverge from other neuroimaging studies and require further replication. The quantity of relevant research has, thus far, been limited and the association between GM and abstinence duration of AUD patients has not yet been systematically reviewed.

Methods: The present research conducted a meta-analysis of voxel-based GM studies in AUD patients published before Jan 2021. The study utilised a whole brain-based d-mapping approach to explore GM changes in AUD patients, and further analysed the relationship between GM deficits, abstinence duration and individual differences.

Results: The current research included 23 studies with a sample size of 846 AUD patients and 878 controls. The dmapping approach identified lower GM in brain regions including the right cingulate gyrus, right insula and left middle frontal gyrus in AUD patients compared to controls. Meta-regression analyses found increasing GM atrophy in the right insula associated with the longer mean abstinence duration of the samples in the studies in our analysis. GM atrophy was also found positively correlated with the mean age of the samples in the right insula, and positively correlated with male ratio in the left middle frontal gyrus.

Conclusions: GM atrophy was found in the cingulate gyrus and insula in AUD patients. These findings align with published meta-analyses, suggesting they are potential deficits for AUD patients. Abstinence duration, age and gender also affect GM atrophy in AUD patients. This research provides some evidence of the underlying neuroanatomical nature of AUD.
\end{abstract}

Keywords: Alcohol use disorders, Abstinence, Grey matter, Meta-analysis, Voxel-based morphometry

\footnotetext{
*Correspondence: litaohx@scu.edu.cn

${ }^{\dagger}$ Lei Li and Hua Yu contributed equally to this work.

${ }^{1}$ Mental Health Center, West China Hospital of Sichuan University, Chengdu, Sichuan, People's Republic of China

${ }^{2}$ Psychiatric Laboratory, State Key Laboratory of Biotherapy, West China Hospital, Sichuan University, Chengdu, Sichuan, People's Republic of China Full list of author information is available at the end of the article
}

(c) The Author(s). 2021 Open Access This article is licensed under a Creative Commons Attribution 4.0 International License, which permits use, sharing, adaptation, distribution and reproduction in any medium or format, as long as you give appropriate credit to the original author(s) and the source, provide a link to the Creative Commons licence, and indicate if changes were made. The images or other third party material in this article are included in the article's Creative Commons licence, unless indicated otherwise in a credit line to the material. If material is not included in the article's Creative Commons licence and your intended use is not permitted by statutory regulation or exceeds the permitted use, you will need to obtain permission directly from the copyright holder. To view a copy of this licence, visit http://creativecommons.org/licenses/by/4.0/. The Creative Commons Public Domain Dedication waiver (http://creativecommons.org/publicdomain/zero/1.0/) applies to the data made available in this article, unless otherwise stated in a credit line to the data. 


\section{Background}

The literature has long framed alcohol use disorders (AUD) as chronic brain diseases [1]. The brain disease model of addiction states that GM atrophy involves in the reward circuit, as well as brain regions relevant to decision-making [2, 3]. More importantly, this abnormality of the reward circuit and decision making would negatively reinforce to craving, which leads to substance abuse eventually being compulsive [4]. The disease model is supported by numerous neuroimaging studies using structural magnetic resonance imaging (MRI) by analysing voxel-based morphometry (VBM). However, the pathology of this disease model requires clarification.

VBM analysis by MRI techniques has provided evidence of GM deficits in cortical areas, including the prefrontal cortex (PFC) [5], anterior cingulate gyrus (ACC) [6], parietal cortex [7], and in subcortical brain regions, including the hippocampus [8], the thalamus, the nucleus accumbens [9] and the amygdala [10] in individuals with AUD. Those brain regions are believed to be involved with decision-making and reward processing. Previous meta-analysis and reviews have suggested that GM is lower in the cingulate gyrus, striatum and insula in AUD patients compared to control samples [11, 12], whereas observed changes in the PFC, dorsal lateral prefrontal cortex, left thalamus and right hippocampus remain inconsistent.

One of the possibilities is that the sample gender ratio may contribute to individual differences in GM volume. It has been reported in some previous studies that females are more vulnerable to the effects of alcohol on GM than males [13]. However, other studies reported that males with AUD had lower GM in the thalamus and putamen compared to their nondrinking peers, whereas females with AUD had greater GM in the thalamus and putamen [14]. The gender ratio in Xiao et al. [11] was not examined, which could have affected findings.

Another possibility posits that the samples age and the age at onset could moderate the rate of GM atrophy. For example, adolescent brains are especially susceptible to the effects of alcohol [15]. It was reported that those who started drinking before the age of 16 are more likely to develop alcohol dependence than those who started after 21 [16]. Alternatively, Sullivan et al. [17] reported a controversial age-alcoholism interaction showing that older individuals with alcohol dependence obtain greater deficits than controls because older brains are more susceptible to alcohol, regardless of the duration of alcohol dependence. Thayer et al. [18] reported that samples under the age of 25 would suffer serious GM atrophy and different brain regions seemed to suffer the effect, where increase of GM atrophy in the left lateral orbitofrontal cortex was correlated with age in the samples over 25 years old. On the other hand, Xiao et al. [11] did not find evidence for any effect of age. The results from these previous studies were therefore inconsistent.

Apart from age and gender, the duration of time after abstinence from alcohol could also potentially affect GM. Recent research reported a sustained compensatory effect on GM in the cingulate gyrus and insular regions, that is, no more significant GM atrophy was found after abstinence from alcohol, suggesting that abstinence from alcohol potentially allows for structural recovery in GM in AUD patients [19]. However, this effect diminished in the pre-cuneus that the GM atrophy reappeared with a longer abstinence duration. In other words, individuals with alcohol dependence could experience corresponding atrophy in GM after abstaining from alcohol. Mann et al. reviewed similar cases in AUD patients [20]. Therefore, the current study will perform metaregression analyses of abstinence duration for AUD to provide comparable replication.

The current research includes newly published studies on AUD (within the past 21 years) and performed an anisotropic effect size-signed differential mapping (AESSDM) for neuroimaging studies. A meta-analysis using the SDM toolbox identified the most consistent brain changes in space with the coordinate information reported in previous studies. We aimed to establish the most consistent brain structural abnormalities of AUD, using all published, whole-brain structural MRI studies that do not bias findings to a priori hypothesised regions. Then, the impact of AUD abstinence and other individual differences on the GM morphology with the latest studies and a larger sample size of AUD patients was examined. From the literature reviewed above, we hypothesised that, firstly, the GM of the AUD group would differ significantly from the control group, and these differences would show consistency with previously published meta results; Secondly, mean abstinence duration in the studies and individual parameters of the samples, such as mean age and gender ratio would have effects on the GM atrophy.

\section{Methods}

\section{Data source}

A systematic and comprehensive search strategy was used to collect studies in PubMed (https://www.ncbi. nlm.nih.gov/pubmed/), CENTER (Cochrane Library) (https://www.cochrane.org/), Embase (www.embase. com), and Google Scholar (http://scholar.google.com/) from Jan 2000 to Jan 2021. DSM-V combines diagnostic criteria for abuse and dependence into a unitary diagnostic category of AUD. This study utilised a combination of the following keywords: (1) voxel-based morphometry; VBM; morphometry; volumetry; grey 
matter; or structural MRI and (2) alcohol dependent; alcoholism; alcohol abuse or alcohol use disorder.

\section{Inclusion/exclusion criteria}

The reference lists of identified studies and relevant theoretical reviews were then manually checked for additional studies.

The inclusion criteria: (1) subjects with alcohol abuse disorder, alcohol dependence disorder or alcohol use disorder that met the DSM-IV-TR or International Statistical Classification of Diseases and Related Health Problems-10th Revision (ICD-10) diagnostic criteria; (2) use of VBM to analyse the GM differences in patients with AUD and control subjects; (3) results of wholebrain GM alterations reported in MNI space; (4) participants aged $\geq 18$, and all participants provided informed consent; (5) thresholds for significance corrected for multiple comparisons; (6) peer reviewed studies; and (7) articles published in English (for quality check purpose).

The exclusion criteria: (1) studies used a region-ofinterest (ROI) or seed voxel-based analysis only; (2) studies included participants aged < 18 years old; (3) studies analysed white matter changes or cortical thickness only; (4) research material comprised review articles, theoretical papers or animal experimental studies; (5) Chinese articles; (6) studies included patients with other comorbid psychiatric disorders; and (7) original coordinates were not reported, and the author did not respond to email inquiries (Fig. 1).

To evaluate the perceived studies' quality, the following criteria was applied: (1) group matching, (2) method of alcohol use diagnosis, (3) group matching on drug use levels, (4) samples size, (5) method used to collect alcohol use history, (6) the use of GM volume or density covariates, (7) MRI machines, smooth kernels, corrected level. Each criterion was independently assessed by two independent reviewers who scored a numerical value from 0 to 5 to evaluate quality. The sum was used in a meta-regression to check the effect of quality.

\section{AES-SDM}

Regional differences in GM between patients with AUD and controls were analysed with AES-SDM (AES-SDM; http://www.sdmproject.com/) [21]. In brief, the main steps of AES-SDM were as follows: Firstly, we extracted peak coordinates to recreate txt file in the included studies, the effect size, such as P- or Z- values for clusters were transformed into t-statistics by SDM online conversion utilities (https://www.sdmproject.com/utilities/ ?show=Statistics). A total of 23 txt files were generated. Secondly, we imported 23 txt files into the SDM software and recreated a sdm_table. Finally, we conducted pre-processing, mean analysis, sensitivity analysis, heterogeneity analyses, meta-regression analysis of mean age, the proportion of males, abstinence duration (days), age at onset and duration of dependence. The studies were given different weights based on number of participants and quality of research, weighted by the square root of the sample size and quality of each study [21].

A whole-brain voxel-based jackknife sensitivity analysis assessed the reproducibility of the results. The analysis was repeated 23 times, with each iteration leaving out a different study. Conclusions can be drawn if differences for a brain region remain significant in more than $75 \%$ of the sensitivity analyses. Variables assessed with the meta-regression analysis included mean age, the percentage of males and abstinence duration as well as age at onset and duration of dependence (less than half of the 23 studies provided this information). Heterogeneity analyses determined if there were significant, unexplained differences between studies. Funnel plots and Egger tests identified conflicting studies and publication bias [22].

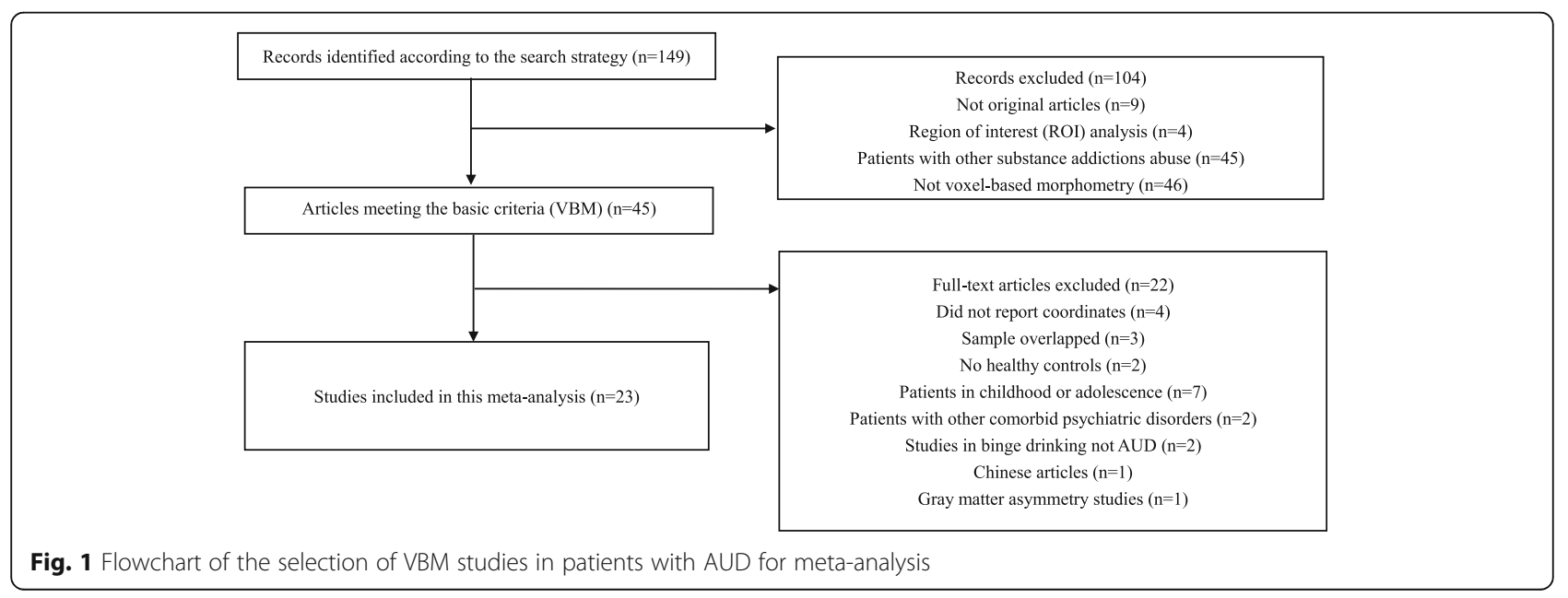




\section{Results}

Demographic and clinical characteristics of patients with AUD and controls

The current research included 23 studies in the metaanalysis based on the search strategy. In total, there were 846 patients with AUD (male $=658$; female $=188$; mean age range: $22.95-53.6$ years) and 878 controls (male $=$ 679; female $=199$; mean age range: $24.63-53.7$ years). Table 1 illustrated the demographic information. No differences were found in age, gender or education between AUD and control groups in each study.

\section{Regional GM differences}

The pooled AES-SDM meta-analysis map revealed significant lower GM in the right cingulate gyrus, right insula and left middle frontal gyrus in AUD patients compared to controls (Fig. 2a). Table 2 shows the peak coordinates and the cluster breakdown. No brain areas with increased GM were observed.

\section{Sensitivity and heterogeneity analyses}

Whole-brain jackknife sensitivity analysis of the findings showed that the main results were highly robust. As shown in Table 3, the whole-brain jackknife sensitivity analysis revealed that lower GM in the right insula and right cingulate gyrus were highly replicable, as this finding was preserved when each study was removed. Lower GM in the left middle frontal gyrus remained significant in 22 out of 23 combinations. Finally, heterogeneity analysis results were reflected by the funnel plot and Egger tests, and the funnel plots did not reveal any publication bias. (see supplementary materials, Fig. S1).

\section{Meta-regression analysis of abstinence, age and gender}

The results of the meta-regression analysis on abstinence duration from the AUD patients revealed an increasing of atrophy in the right insula (MNI coordinate: 44, 10, 4; 55 peak voxels; SDM z $=-4.371 ; p=0.0015$ ) with the longer mean abstinence duration of the samples (Fig. 2b). GM in right insula (MNI coordinate: $54,-2,8 ; 532$ peak voxels; SDM z $=-4.243 ; p=\sim .000$ ) yields a significant negative correlation with the average age of the samples (Fig. 2c). There was a negative association between the male ratio of the studies and GM in the left middle frontal gyrus (MNI coordinate: $-28,38,32 ; 11$ peak voxels; SDM z $=-2.682 ; p=0.002$ ) (Fig. $2 d$ ). Moreover, exploratory meta-regression analysis suggests that the age at onset and duration of dependence in AUD patients was negatively associated with GM volume (supplementary materials, Fig. S2).

\section{Discussion}

The current quantitative meta-analysis of VBM studies included 23 original studies which demonstrated GM reduction in AUD patients with a large samples size. The results showed lower GM in the right cingulate gyrus, right insula and the left middle frontal gyrus in AUD patients compared with controls, which were highly replicated with previously published metaanalysis. Jackknife sensitivity analyses showed that these results were consistent and robust [12]. Then, we found that GM atrophy increased with longer mean duration of abstinence of the samples in the studies. Finally, we found that GM atrophy increased with mean age and the age onset in the samples, and the male ratio of the samples were more sensitive to the harmful effects of alcohol consumption.

\section{GM atrophy in the ACC, insula and middle frontal gyrus}

The results revealed a significant GM atrophy in the ACC and right insula in AUD patients compared to controls, which aligns with previously published reviews [11, 12]. The ACC is related to a range of cognitive functions, including impulse control and assessment of responses and behaviour [23, 24]. Craving for alcohol in AUD patients is related to abnormal brain circuits projected from the ACC to the nucleus accumbens, and GM volume changes may form the structural basis of this [25]. In the healthy controls, inhibitory control was found to be associated with activation in the ACC [26]. However, in healthy adults with a family history of alcoholism, higher impulsivity was associated with lower activation in the ACC [27]. Compared to health controls, smaller GM volume in the ACC was found in alcohol users with higher impulsivity [28]. However, the current studies included in our meta-analysis are not longitudinally designed, and we cannot make a firm conclusion about the causal relationship between GM atrophy and alcohol consumption.

Apart from the ACC, the insula is also an important hub for transmitting salient information to the prefrontal cortices involved in decision-making [29], to the limbic system involved in emotional responses [30] and to the brain reward system via the ventral striatum involved in substance abuse [31]. Functional brain imaging studies have revealed a correlation between insula activity and cravings for alcohol and drugs [32]. The current study reported consistent results: GM in the right insula was lower in the AUD group compared with the controls, replicating previous results.

The current study also demonstrated brain changes not observed in the two previous meta-analyses [11, 12]. The GM in the left middle frontal gyrus in AUD patients were lower than that in the controls. The middle frontal gyrus is thought to play an important role in cognitive functions, including working memory capacity and fluid intelligence [33]. Yang et al. [12] found that shrinkage of GM in the left middle frontal gyrus correlates to lifetime 


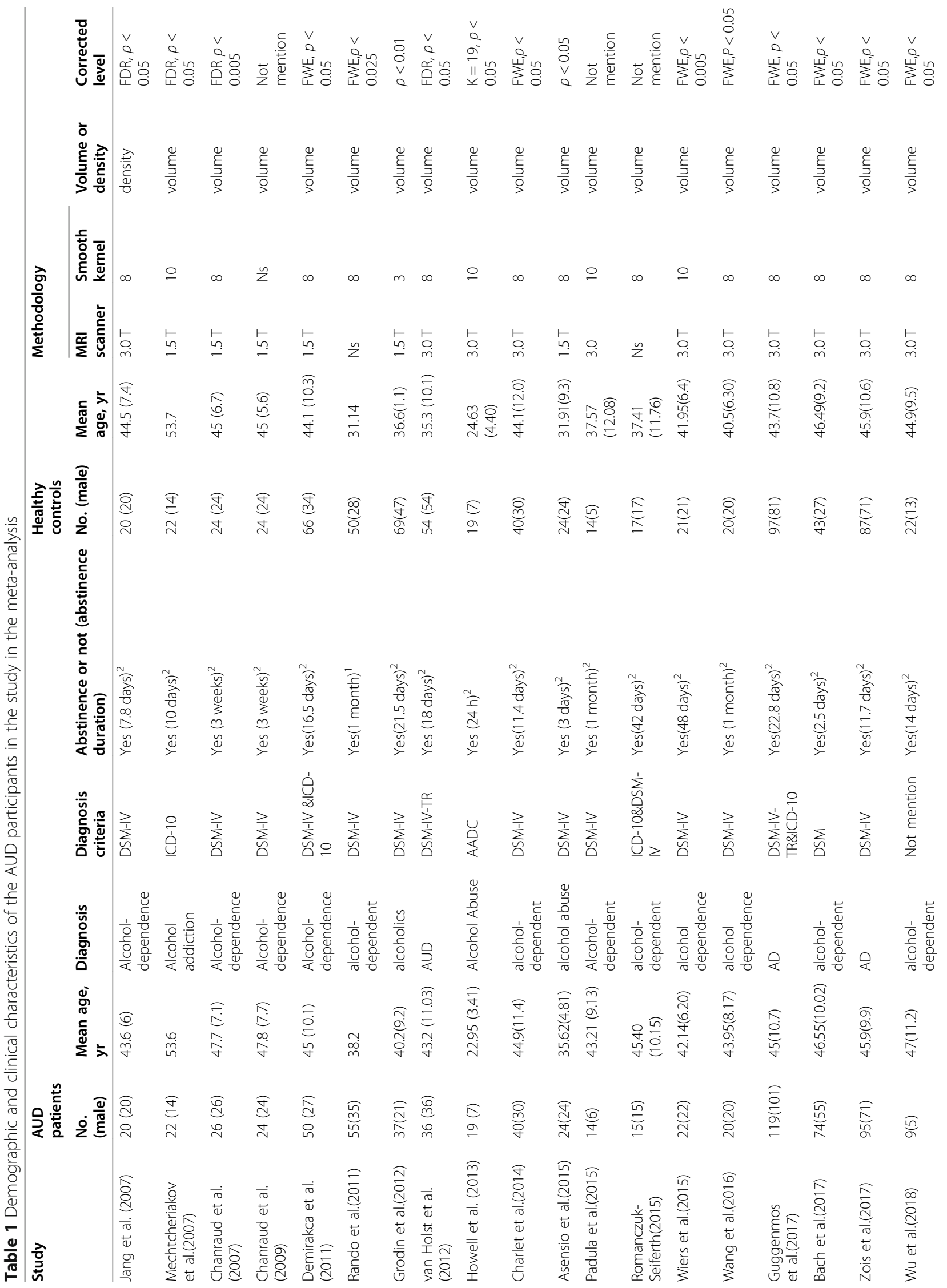




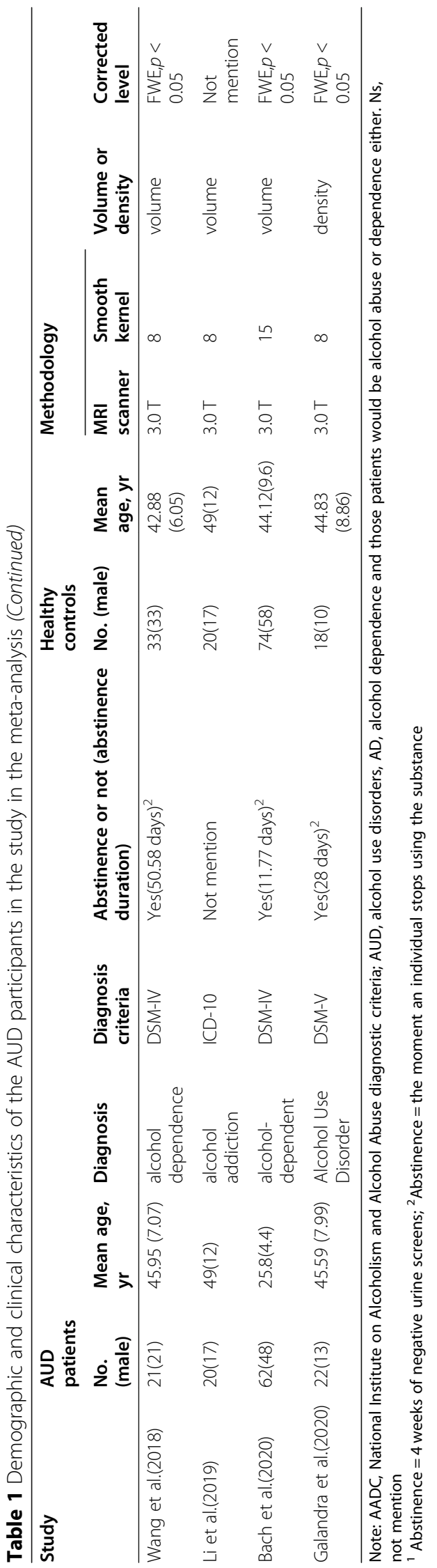


(a)

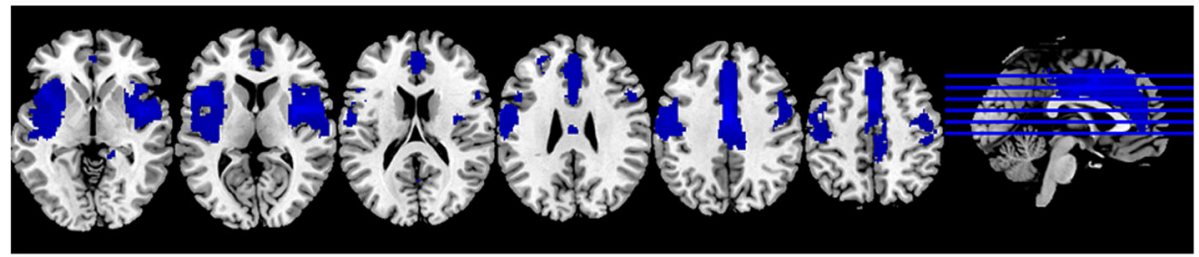

(b)

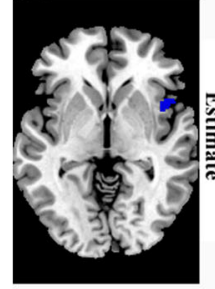

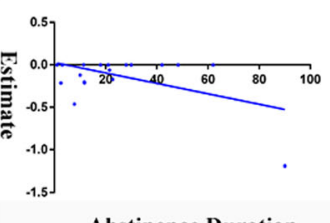

Abstinence Duration (c)

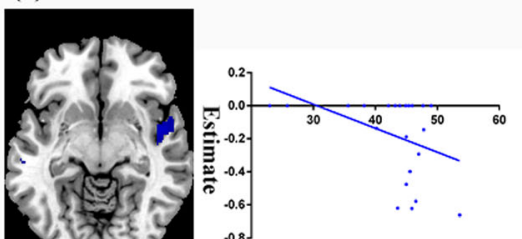

Mean Age

(d)
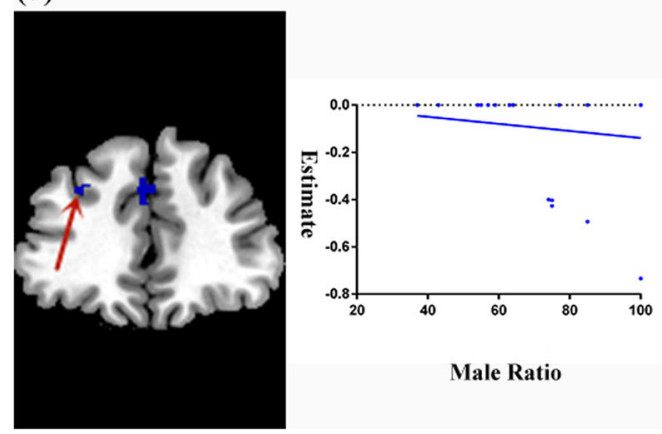

Male Ratio

Fig. 2 Meta-analysis results. a Regions showed lower GM in AUD patients than controls. $\mathbf{b}$ Meta-regression analysis indicated that GM in the right insula was significantly negatively associated with duration of abstinence in AUD patients. c Meta-regression analysis indicated that GM in the right insula was significantly negatively associated with mean age in AUD patients. $\mathbf{d}$ Meta-regression analysis showed that GM in the left middle frontal gyrus was significantly negatively associated with male ratio in AUD patients. Blue colour represents GM reduction

Table 2 Lower grey matter in patients with AUD compared with controls in the meta-analysis

\begin{tabular}{|c|c|c|c|c|c|}
\hline Anatomical regions & $\begin{array}{l}\text { MNI coordinates } \\
x, y, z\end{array}$ & $\begin{array}{l}\text { SDM } \\
\text { value }\end{array}$ & $p$ value & $\begin{array}{l}\text { Number of } \\
\text { voxels }\end{array}$ & Breakdown \\
\hline \multicolumn{6}{|l|}{ AUD $<$ controls } \\
\hline $\begin{array}{l}\text { Right median cingulate / paracingulate } \\
\text { gyri (BA24) }\end{array}$ & $2,6,42$ & -4.438 & $\sim 0$ & 249 & $\begin{array}{l}\text { Right median cingulate / paracingulate } \\
\text { gyri, BA24 } \\
\text { Left median cingulate / paracingulate } \\
\text { gyri, BA24 }\end{array}$ \\
\hline Right insula, BA 48 & $40,0,4$ & -4.581 & $0.000010^{* * * * *}$ & 3641 & $\begin{array}{l}\text { Right insula, BA } 48 \\
\text { Right rolandic operculum, BA } 48 \\
\text { Right inferior frontal gyrus, opercular } \\
\text { part, BA } 44,48 \\
\text { Right precentral gyrus, BA } 4,6\end{array}$ \\
\hline Left middle frontal gyrus, BA 46 & $-28,42,30$ & -3.573 & $0.00015^{* * * *}$ & 106 & $\begin{array}{l}\text { Left middle frontal gyrus, BA } 46 \\
\text { Left middle frontal gyrus }\end{array}$ \\
\hline
\end{tabular}


Table 3 Regions of grey matter differences in AUD patients compared with controls for sensitivity analyses

\begin{tabular}{llllll}
\hline MNI coordinate & SDM-Z & P value & Voxels & Clusters & Jackknife sensitivity analysis \\
\hline $2,6,42$ & -4.438 & $\sim 0$ & 249 & right cingulate & $23 / 23$ \\
$40,0,4$ & -4.581 & $0.000010^{* * * * *}$ & 3641 & Right insula & $23 / 23$ \\
$-28,42,30$ & -3.573 & $0.00015^{* * * *}$ & 106 & Left middle frontal gyrus & $22 / 23$ \\
\hline
\end{tabular}

AUD = Alcohol abuse disorders; $\mathrm{MNI}=$ Montreal Neurological Institute; SDM = signed differential mapping; $\mathrm{BA}=\mathrm{Brodmann}$ area; ${ }^{* * * *} p<0.00001 ;{ }^{* * * *} p<0.0001$

alcohol consumption. Previous studies reported that the prefrontal function of alcohol users experiences gradually damage, which may be related to an increase in alcohol consumption, which is likely to promote increased craving and weaken behavioural control, thereby contributing to further alcohol consumption $[34,35]$.

We replicated previous meta-analysis findings in the ACC and right insula, which identified deficits of GM in the ACC and insula as potential neuroanatomical diagnostic biomarkers in AUD patients. However, GM loss in parietal, temporal and subcortical brain regions was not replicated. One possible explanation is that, with our increased sample size, the observed group effect disappeared, suggesting that GM deficits of these brain areas do not represent stable markers of AUD. Another possible explanation posits that, in the current study, we failed to control whether patients had received effective treatment as drugs may influence these brain regions. A previous animal study in rats found that naltrexone treatment during early abstinence resulted in subtle brain changes potentially distinguishable from non-treated abstinent brains, suggesting the existence of an intermediate state associated with brain recovery from alcohol exposure induced by medication [36]. Future studies should consider drug treatment effects on GM volume.

\section{The persistent of GM atrophy after abstinence}

The current meta-regression analysis found that the right insula shows persistent GM reduction in AUD patients with increasing abstinence duration of the samples, which is comparable with findings in previous studies [37, 38]. However, other studies reported diverse results [39], where the frontal, temporal and insular regions remain no different from controls after abstinence from opioids. One key difference may be the treatment received by patients. The current study, as well as previous studies [37, 38], failed to control whether samples have received any treatment, what type of treatment or the intensity, because this information was unavailable. According to Wollman and colleagues' 2017 study [39], all participants received drug substitution therapy (methadone) to achieve abstinence and no GM atrophy was observed post treatment. Therefore, the abstinence in the current study should not represent as recovery but only abstinence from alcohol consumption at a behavioural level. In other words, it remains possible that participants never actually achieved abstinence. Another interpretation of our results is that the GM atrophy of insula in AUD groups persists even with the absence of addictive behaviour. Brain atrophy in AUD patients did not reach the equivalent level to controls after abstinence [20]. Based on the evidence in opioid dependence, hypothetically, the GM volume may stay constant once it recovers to normal at a neurological level [39, 40]. Since the GM volume of the insula did not eventually recover to a normal neurological level in the current study, it may be inferred that this insula GM atrophy would continue to deteriorate. As a result, we suggest further studies should consider abstinence in more detail.

Another potential interpretation for the damage in the insula is that the GM atrophy caused "the sense of abstinence" rather than caused by abstinence. The insula plays an important role in the craving of addictive behaviours and it might also facilitate withdrawal symptoms by translating the physiological state of withdrawal into dysphonia [41]. This is only one of many functions of the insula and the GM atrophy in the insula limits its capacity to perform [42], such that the translation from the urge of neurotransmitters into craving is limited. There is empirical evidence in nicotine dependence to support the theory that the GM atrophy in the insula area has been found negatively correlated with tobacco craving $[43,44]$. However, this theory has not been directly examined in other types of substance abuse, and the current study observed a comparable pattern, that is, the abstinence duration positively correlated with GM atrophy in the insula. Hypothetically, this GM reduction limited the capacity of the insula and reduced the craving of alcohol consumption to maintain their abstinence.

\section{Impact of age and gender on GM atrophy}

The current study demonstrates a significant negative association between GM in the insula and average the age of the samples, suggesting that any GM deficit would be stronger in older AUD patients. Several previous reviews have illustrated the same interaction between age and GM atrophy [17, 18]. A study by Sullivan et al. [17] assessed the impact of age independently for control and alcoholism groups, and observed that the alcoholism 
group showed effects of age in the insula, while the control group did not. Additionally, our study was consistent with the results reported by Thayer et al. [18]. In their study, insula deficits caused by alcohol were negatively associated with increased age. However, some evidence points out that GM volume would decrease due to ageing regardless of external interference [45], and consuming alcohol would reinforce the loss of GM to eventually present an alcohol-age interaction $[17,46]$.

Meta-regression analysis illustrated an increase of GM atrophy in right cingulate gyrus with age at onset in AUD patients. Other studies found that age at onset plays an important role to GM atrophy in AUD patients. For example, those who started drinking before 14 years old were more likely to develop alcohol dependence than those who started drinking after 21 [16]. Moreover, less activation of the cingulate gyrus was also found in adolescents who started drinking after 14 years old compared with no or minimal alcohol users [47]. Our results were different from these two but not contradictory because the previous studies primarily focused on adolescent-onset patients, as the adolescent are more susceptible to the effects of alcohol [15]. Our samples are adult-onset patients, which means the relationship between age at onset and GM atrophy in AUD patients was possibly not absolute linear and requires further investigation.

Additionally, the GM of middle frontal gyrus decreased when the dependence duration increases in AUD patients, which was consistent with the previous analysis that increased alcohol consumption duration will lead to neuronal loss and accelerate with brain atrophy $[48,49]$. Our results suggested that further research should include this information as these might have impact on brain structure. Considering that the age at the time of assessment was correlated with age at onset in alcohol studies, the brain region affected by age and age onset might also be affected by the duration of illness, that longer duration was related to higher GM atrophy. However, we found the brain regions are affected by age, age onset and illness duration are different. It is important to point out that the results of age at onset and duration of dependence should be interpreted with cautious, where less than half of the 23 studies had reported the information to be included in the metaregression analysis, which makes the results cannot be generalised to the whole samples. To address this point, we suggest future studies to report the details of the illness duration.

Finally, the present research revealed a negative association between GM in the left middle frontal gyrus and the gender of the samples, showing that males are more vulnerable to the harmful effects of alcohol on GM than females. Other studies have reported gender differences in GM proportion in intracranial content [50] and agegender interactions in the hippocampus [45]. Though our study was not consistent with the results reported by Hommer et al. [50], which included a more balanced gender ratio ( male $=43$; female $=36$ ) in their analysis compared to the current study (male $=658$; female $=$ 188). Therefore, the lack of female cases in the present work potentially obscured the impact of gender on GM atrophy.

\section{Limitation}

There were several limitations for the current study. Firstly, the analysed data comprised the coordinates in the published studies rather than the original data which may result in less accurate findings [51]. Secondly, the heterogeneity of methodologies in VBM studies including MRI machines, smooth kernels and corrected level, for example FWER or FDR, might represent a critical factor and couldn't be controlled for. However, we examine these potential confounders by using quality of the study, which did not affect our results (supplementary material Fig. S3). Thirdly, the current study could not control whether participants had previously smoked, concurrent drinking [52], cardiovascular disease [53]. The studies did not record this information but may contribute to GM thinning in AUD patients. Fourthly, alcohol use disorders and other psychiatric disorders are commonly co-occur co-morbidities, our current results could not be generalised to comorbidity population (Alcohol Use Disorder and Co-Occurring Mental Health Conditions). We suggested that future studies of this field should provide a more detailed comparison between patients with and without comorbidity.

\section{Conclusions}

The current research reported partially consistent results with previous reviews. It reported that GM was lower in the insula, cingulate gyrus, and middle frontal gyrus in the AUD group compared to controls. Moreover, there was lower GM in the insula with increased mean abstinence duration of the samples in the studies. GM atrophy deteriorated more with an increased mean age in our samples, and the males of the samples were reported to be more sensitive to the GM atrophy among AUD patients. The current study included studies which reported their treatments and others did not, making it unable to fairly determine the impact of treatment on GM atrophy. The future research should consider reporting their treatment with more details and placing heterogeneous analysis on the effect of treatment.

\section{Abbreviations}

GM: grey matter; AUD: alcohol use disorders; MRI: magnetic resonance imaging; VBM: voxel-based morphometry; PFC: prefrontal cortex; 
ACC: anterior cingulate gyrus; AES-SDM: anisotropic effect size-signed differential mapping

\section{Supplementary Information}

The online version contains supplementary material available at https://doi. org/10.1186/s12888-021-03244-9.

Additional file 1 Fig. S1. Funnel plots were used to assess publication bias. The funnel plot did not reveal any publication bias as Egger test > 0.05. Fig. S2. Meta-regression analysis results. a GM in right cingulate gyrus was significantly negatively associated with age of onset in AUD patients. b GM in left middle frontal gyrus was significantly negatively associated with duration of illness in AUD patients. Blue color represents GM reduction. Fig. S3. Region differences in GM in AUD patients in subgroup meta-analysis of MRI machines (a) and smooth kernels (b). Blue color represents GM reduction. Fig. S4. Visualize of the samples sizes included in our meta-analysis. Table S1. Each study described abstinence duration in AUD patients.

\section{Acknowledgments}

We would like to thank all the authors who found those original studies and detailed data. We would specially thank Dr. Lorna Hardy from the Department of Psychology, College of Life and Environmental Science at the University of Exeter for her support in academic English writing.

\section{Authors' contributions}

$L L, H Y$ and $T L$ were responsible for the study concept and design. $L L, H Y, Y L$, $Y M, X L, C Z, S L, M L$ collected the data, analysed data, interpreted the results. $\mathrm{LL}$ and $\mathrm{HY}$ wrote the manuscript. WG, WQ, WD, XM, JC and TL provided critical revision of the manuscript. All authors read and approved the final manuscript.

\section{Funding}

This work was partly supported by National Key Research and Development Program of the Ministry of Science and Technology of China

(2016YFC0904300), National Nature Science Foundation of China Key Project (81630030, 81920108018 and 8157130); Department of Science and Technology of Sichuan Provincial Government (2019YFS0153) and 1.3.5 Project for Disciplines of Excellence, West China Hospital, Sichuan University (ZY2016203, ZY2016103, ZYGD200004, 2019HXFH026). The funding body had no further role in the design of the study and collection, analysis, and interpretation of data and in writing the manuscript.

\section{Availability of data and materials}

The data and materials during this study can be made available in this article.

\section{Declarations}

Ethics approval and consent to participate

Not applicable.

\section{Consent for publication}

Not applicable.

\section{Competing interests}

No conflicts of interest declared by any author.

\section{Author details}

'Mental Health Center, West China Hospital of Sichuan University, Chengdu, Sichuan, People's Republic of China. ${ }^{2}$ Psychiatric Laboratory, State Key Laboratory of Biotherapy, West China Hospital, Sichuan University, Chengdu, Sichuan, People's Republic of China. ${ }^{3}$ Brain Research Center, West China Hospital of Sichuan University, Chengdu, China. ${ }^{4}$ Department of Psychology, College of Life and Environmental Science, University of Exeter, Exeter, UK.
Received: 23 September 2020 Accepted: 28 April 2021

Published online: 11 May 2021

\section{References}

1. Leshner Al. Addiction is a brain disease, and it matters. Science. 1997; 278(5335):45-7. https://doi.org/10.1126/science.278.5335.45.

2. Koob GF. The neurobiology of addiction: a neuroadaptational view relevant for diagnosis. Addiction. 2006;101(Suppl 1):23-30. https://doi.org/10.1111/ j.1360-0443.2006.01586.x.

3. Wise RA. Dopamine and reward: the anhedonia hypothesis 30 years on. Neurotox Res. 2008;14(2-3):169-83. https://doi.org/10.1007/BF03033808.

4. Volkow ND, Swanson JM, Evins AE, DeLisi LE, Meier MH, Gonzalez R, et al. Effects of Cannabis use on human behavior, including cognition, motivation, and psychosis: a review. JAMA Psychiatr. 2016;73(3):292-7. https://doi.org/10.1001/jamapsychiatry.2015.3278.

5. Gröpper S, Spengler S, Stuke H, Gawron CK, Parnack J, Gutwinski S, et al. Behavioral impulsivity mediates the relationship between decreased frontal gray matter volume and harmful alcohol drinking: a voxel-based morphometry study. J Psychiatr Res. 2016;83(Complete):16-23.

6. Zois E, Vollstädt-Klein S, Hoffmann S, Reinhard I, Charlet K, Beck A, et al. Orbitofrontal structural markers of negative affect in alcohol dependence and their associations with heavy relapse-risk at 6 months post-treatment. Eur Psychiatr. 2017:46:16-22. https://doi.org/10.1016/j.eurpsy.2017.07.013.

7. Fein $G$, Shimotsu R, Chu R, Barakos J. Parietal gray matter volume loss is related to spatial processing deficits in long-term abstinent alcoholic men. Alcohol Clin Exp Res. 2009;33(10):1806-14. https://doi.org/10.1111/j.1530-02 77.2009.01019.x

8. Lee J, Im SJ, Lee SG, Stadlin A, Son JW, Shin CJ, et al. Volume of hippocampal subfields in patients with alcohol dependence. Psychiatr Res Neuroimaging. 2016;258:16-22. https://doi.org/10.1016/j.pscychresns.2016.1 0.009 .

9. Shim JH, Kim YT, Kim S, Baek HM. Volumetric reductions of subcortical structures and their localizations in alcohol-dependent patients. Front Neurol. 2019;10:247. https://doi.org/10.3389/fneur.2019.00247.

10. Benegal V, Antony G, Venkatasubramanian G, Jayakumar PN. Gray matter volume abnormalities and externalizing symptoms in subjects at high risk for alcohol dependence. Addict Biol. 2007;12(1):122-32. https://doi.org/1 0.1111/j.1369-1600.2006.00043.x.

11. Xiao P, Dai Z, Zhong J, Zhu Y, Shi H, Pan P. Regional gray matter deficits in alcohol dependence: a meta-analysis of voxel-based morphometry studies. Drug Alcohol Depend. 2015;153:22-8. https://doi.org/10.1016/j.drugalcdep.2 015.05.030.

12. Yang $X$, Tian F, Zhang H, Zeng J, Chen T, Wang S, et al. Cortical and subcortical gray matter shrinkage in alcohol-use disorders: a voxel-based meta-analysis. Neurosci Biobehav Rev. 2016;66:92-103. https://doi.org/10.101 6/j.neubiorev.2016.03.034.

13. Rosenbloom M, Sullivan EV, Pfefferbaum A. Using magnetic resonance imaging and diffusion tensor imaging to assess brain damage in alcoholics. Alcohol Res Health. 2003;27(2):146-52.

14. Fein G, Greenstein D, Cardenas VA, Cuzen NL, Fouche JP, Ferrett $H$, et al Cortical and subcortical volumes in adolescents with alcohol dependence but without substance or psychiatric comorbidities. Psychiatr Res. 2013; 214(1):1-8. https://doi.org/10.1016/j.pscychresns.2013.06.001.

15. Crews F, He J, Hodge C. Adolescent cortical development: a critical period of vulnerability for addiction. Pharmacol Biochem Behav. 2007;86(2):189-99. https://doi.org/10.1016/j.pbb.2006.12.001.

16. Hingson RW, Heeren T, Winter MR. Age at drinking onset and alcohol dependence: age at onset, duration, and severity. Arch Pediatr Adolesc Med. 2006;160(7):739-46. https://doi.org/10.1001/archpedi.160.7.739.

17. Sullivan EV, Zahr NM, Sassoon SA, Thompson WK, Kwon D, Pohl KM, et al. The role of aging, drug dependence, and hepatitis C comorbidity in alcoholism cortical compromise. JAMA Psychiatr. 2018;75(5):474-83. https:// doi.org/10.1001/jamapsychiatry.2018.0021.

18. Thayer RE, Hagerty SL, Sabbineni A, Claus ED, Hutchison KE, Weiland BJ. Negative and interactive effects of sex, aging, and alcohol abuse on gray matter morphometry. Hum Brain Mapp. 2016;37(6):2276-92. https://doi. org/10.1002/hbm.23172.

19. Demirakca T, Ende G, Kämmerer N, Welzel-Marquez H, Hermann D, Heinz A, et al. Effects of alcoholism and continued abstinence on brain volumes in both genders. Alcohol Clin Exp Res. 2011;35(9):1678-85. 
20. Mann K, Ackermann K, Croissant B, Mundle G, Nakovics H, Diehl A. Neuroimaging of gender differences in alcohol dependence: are women more vulnerable? Alcohol Clin Exp Res. 2005;29(5):896-901. https://doi.org/1 0.1097/01.ALC.0000164376.69978.6B.

21. Radua J, Rubia K, Canales-Rodriguez EJ, Pomarol-Clotet E, Fusar-Poli P, Mataix-Cols D. Anisotropic kernels for coordinate-based meta-analyses of neuroimaging studies. Front Psychiatr. 2014;5:13.

22. Carlisi CO, Norman LJ, Lukito SS, Radua J, Mataix-Cols D, Rubia K. Comparative multimodal Meta-analysis of structural and functional brain abnormalities in autism Spectrum disorder and obsessive-compulsive disorder. Biol Psychiatry. 2017;82(2):83-102. https://doi.org/10.1016/j. biopsych.2016.10.006.

23. Claus ED, Ewing SW. Feldstein, Filbey FM, Hutchison KE: behavioral control in alcohol use disorders: relationships with severity. J Stud Alcohol Drugs. 2013;74(1):141-51. https://doi.org/10.15288/jsad.2013.74.141.

24. Crews FT, Boettiger CA. Impulsivity, frontal lobes and risk for addiction. Pharmacol Biochem Behav. 2009;93(3):237-47. https://doi.org/10.1016/j. pbb.2009.04.018.

25. Stone JM, Day F, Tsagaraki H, Valli I, McLean MA, Lythgoe DJ, et al. Glutamate dysfunction in people with prodromal symptoms of psychosis: relationship to gray matter volume. Biol Psychiatry. 2009;66(6):533-9. https:// doi.org/10.1016/j.biopsych.2009.05.006.

26. Karoly HC, Weiland BJ, Sabbineni A, Hutchison KE. Preliminary functional MRI results from a combined stop-signal alcohol-cue task. J Stud Alcohol Drugs. 2014;75(4):664-73. https://doi.org/10.15288/jsad.2014.75. 664.

27. Cohen-Gilbert JE, Sneider JT, Crowley DJ, Rosso IM, Jensen JE, Silveri MM. Impact of family history of alcoholism on glutamine/glutamate ratio in anterior cingulate cortex in substance-naive adolescents. Dev Cogn Neurosci. 2015;16:147-54. https://doi.org/10.1016/j.dcn.2015.04.005.

28. Grodin EN, Cortes CR, Spagnolo PA, Momenan R. Structural deficits in salience network regions are associated with increased impulsivity and compulsivity in alcohol dependence. Drug Alcohol Depend. 2017;179:100-8. https://doi.org/10.1016/j.drugalcdep.2017.06.014.

29. Everitt BJ, Robbins TW. Neural systems of reinforcement for drug addiction: from actions to habits to compulsion. Nat Neurosci. 2005;8(11):1481-9. https://doi.org/10.1038/nn1579.

30. Mesulam MM, Mufson EJ: The insula of Reil in man and monkey. 1985, The Insula of Reil in Man and Monkey.

31. Gardini S, Venneri A. Reduced grey matter in the posterior insula as a structural vulnerability or diathesis to addiction. Brain Res Bull. 2012;87(2-3): 205-11. https://doi.org/10.1016/j.brainresbull.2011.11.021.

32. Naqvi $\mathrm{NH}$, Bechara $\mathrm{A}$. The insula and drug addiction: an interoceptive view of pleasure, urges, and decision-making. Brain Struct Funct. 2010;214(5-6): 435-50. https://doi.org/10.1007/s00429-010-0268-7.

33. Colom R, Burgaleta M, Roman FJ, Karama S, Alvarez-Linera J, Abad FJ, et al. Neuroanatomic overlap between intelligence and cognitive factors: morphometry methods provide support for the key role of the frontal lobes. Neurolmage. 2013;72:143-52. https://doi.org/10.1016/j.neuroimage.2 013.01.032.

34. Gang C, Carlson VC, Cuzon JW, Anne B, Andreas H, Dorit R, et al. Striatal involvement in human alcoholism and alcohol consumption, and withdrawal in animal models. Alcohol Clin Exp Res. 2011;35(10):1739-48.

35. Volkow ND, Gene-Jack W, Frank T, Fowler JS, Jean L, Millard J, et al. Profound decreases in dopamine release in striatum in detoxified alcoholics: possible orbitofrontal involvement. J Neurosci. 2007;27(46):12700-6. https:// doi.org/10.1523/JNEUROSCI.3371-07.2007.

36. Cosa A, Moreno A, Pacheco-Torres J, Ciccocioppo R, Hyytiä P, Sommer WH, et al. Multi-modal MRI classifiers identify excessive alcohol consumption and treatment effects in the brain. Addict Biol. 2017;22(5):1459-72. https://doi. org/10.1111/adb.12418.

37. Mueller SG, Meyerhoff DJ. The gray matter structural connectome and its relationship to alcohol relapse: Reconnecting for recovery. Addict Biol. 2019; 26(1):e12860.

38. Zou X, Durazzo TC, Meyerhoff DJ. Regional brain volume changes in alcohol-dependent individuals during short-term and long-term abstinence. Alcohol Clin Exp Res. 2018;42(6):1062-72. https://doi.org/10.1111/acer.13757.

39. Wollman SC, Alhassoon OM, Hall MG, Stern MJ, Connors EJ, Kimmel CL, et al. Gray matter abnormalities in opioid-dependent patients: a neuroimaging meta-analysis. Am J Drug Alcohol Abuse. 2017:43(5):505-17. https://doi.org/10.1080/00952990.2016.1245312.
40. Zahr NM, Pfefferbaum A. Alcohol's effects on the brain: neuroimaging results in humans and animal models. Alcohol Res. 2017:38(2):183-206.

41. Naqvi NH, Bechara A. The hidden island of addiction: the insula. Trends Neurosci. 2009;32(1):56-67. https://doi.org/10.1016/j.tins.2008.09.009.

42. Regner MF, Tregellas J, Kluger B, Wylie K, Gowin JL, Tanabe J. The insula in nicotine use disorder: functional neuroimaging and implications for neuromodulation. Neurosci Biobehav Rev. 2019;103:414-24. https://doi.org/1 0.1016/j.neubiorev.2019.06.002.

43. Naqvi NH, Rudrauf D, Damasio H, Bechara A. Damage to the insula disrupts addiction to cigarette smoking. Science. 2007;315(5811):531-4. https://doi. org/10.1126/science.1135926.

44. Abdolahi A, Williams GC, Benesch CG, Wang HZ, Spitzer EM, Scott BE, et al. Damage to the insula leads to decreased nicotine withdrawal during abstinence. Addiction. 2015;110(12):1994-2003. https://doi.org/10.1111/a dd.13061.

45. Taki Y, Thyreau B, Kinomura S, Sato K, Goto R, Wu K, et al. A longitudinal study of age- and gender-related annual rate of volume changes in regional gray matter in healthy adults. Hum Brain Mapp. 2013;34(9):2292301. https://doi.org/10.1002/hbm.22067.

46. Pfefferbaum A, Lim KO, Zipursky RB, Mathalon DH, Rosenbloom MJ, Lane B, et al. Brain gray and white matter volume loss accelerates with aging in chronic alcoholics: a quantitative MRI study. Alcohol Clin Exp Res. 1992; 16(6):1078-89. https://doi.org/10.1111/j.1530-0277.1992.tb00702.x.

47. Norman AL, Pulido C, Squeglia LM, Spadoni AD, Paulus MP, Tapert SF. Neural activation during inhibition predicts initiation of substance use in adolescence. Drug Alcohol Depend. 2011;119(3):216-23. https://doi.org/10.1 016/j.drugalcdep.2011.06.019.

48. Kril JJ, Halliday GM, Svoboda MD, Cartwright $H$. The cerebral cortex is damaged in chronic alcoholics. Neuroscience. 1997;79(4):983-98. https://doi. org/10.1016/S0306-4522(97)00083-3.

49. Cardenas VA, Studholme C, Gazdzinski S, Durazzo TC, Meyerhoff DJ. Deformation-based morphometry of brain changes in alcohol dependence and abstinence. Neurolmage. 2007;34(3):879-87. https://doi.org/10.1016/j. neuroimage.2006.10.015

50. Hommer D, Momenan R, Kaiser E, Rawlings R. Evidence for a gender-related effect of alcoholism on brain volumes. Am J Psychiatry. 2001;158(2):198-204. https://doi.org/10.1176/appi.ajp.158.2.198.

51. Lim L, Radua J, Rubia K. Gray matter abnormalities in childhood maltreatment: a voxel-wise meta-analysis. Am J Psychiatry. 2014;171(8):85463. https://doi.org/10.1176/appi.ajp.2014.13101427.

52. Rolland B, Dricot L, Creupelandt C, Maurage P, De Timary P. Respective influence of current alcohol consumption and duration of heavy drinking on brain morphological alterations in alcohol use disorder. Addict Biol. 2020;25(2):e12751. https://doi.org/10.1111/adb.12751.

53. Durazzo TC, Nguyen LC, Meyerhoff DJ. Medical conditions linked to atherosclerosis are associated with magnified cortical thinning in individuals with alcohol use disorders. Alcohol Alcohol. 2020;55(4):382-90. https://doi. org/10.1093/alcalc/agaa034.

\section{Publisher's Note}

Springer Nature remains neutral with regard to jurisdictional claims in published maps and institutional affiliations.

Ready to submit your research? Choose BMC and benefit from:

- fast, convenient online submission

- thorough peer review by experienced researchers in your field

- rapid publication on acceptance

- support for research data, including large and complex data types

- gold Open Access which fosters wider collaboration and increased citations

- maximum visibility for your research: over $100 \mathrm{M}$ website views per year

At BMC, research is always in progress.

Learn more biomedcentral.com/submissions 